\title{
Time-Kill Kinetics of Rezafungin (CD101) in Vagina-Simulative Medium for Fluconazole-Susceptible and Fluconazole-Resistant Candida albicans and Non-albicans Candida Species
}

\author{
Jeffrey B. Locke $\mathbb{D}$, Amanda L. Almaguer, Joanna L. Donatelli, and Ken F. Bartizal \\ Cidara Therapeutics, Inc., San Diego, CA, USA \\ Correspondence should be addressed to Jeffrey B. Locke; jlocke@cidara.com
}

Received 12 July 2017; Accepted 17 January 2018; Published 22 February 2018

Academic Editor: Bryan Larsen

Copyright (C) 2018 Jeffrey B. Locke et al. This is an open access article distributed under the Creative Commons Attribution License, which permits unrestricted use, distribution, and reproduction in any medium, provided the original work is properly cited.

Background. While echinocandins demonstrate excellent efficacy against Candida species in disseminated infections and demonstrate potent minimal inhibitory concentration (MIC) values under standard susceptibility testing conditions, investigation under conditions relevant to the vaginal environment was needed. We assessed the antifungal activity and time-kill kinetics of the novel echinocandin rezafungin (formerly CD101) under such conditions, against Candida species relevant to vulvovaginal candidiasis (VVC). Methods. Susceptibility testing of fluconazole-susceptible and fluconazole-resistant C. albicans, C. glabrata, C. tropicalis, C. parapsilosis, and C. krusei was performed in RPMI at pH 7.0 and in vagina-simulative medium (VSM) at pH $4.2 \mathrm{for}$ topical rezafungin, terconazole, fluconazole, and amphotericin B. Time-kill kinetics were evaluated for rezafungin and terconazole at 2, 8, 32, and $128 \mu \mathrm{g} / \mathrm{ml}$ over 72 hours. Results. Rezafungin MIC values were the same or 2-fold higher in VSM/pH 4.2 versus $\mathrm{RPMI} / \mathrm{pH}$ 7.0. Some C. albicans terconazole MIC values were lower, but most were significantly higher in VSM than in RPMI. Rezafungin was fungicidal against 11/14 strains and near-fungicidal against the others. Terconazole $(128 \mu \mathrm{g} / \mathrm{ml})$ was fungicidal against C. krusei and near-fungicidal against susceptible C. parapsilosis but fungistatic versus all other strains evaluated. Conclusion. Rezafungin retained anti-Candida activity and fungicidal activity under in vitro conditions relevant to VVC.

\section{Introduction}

Vulvovaginal candidiasis (VVC) affects most women at least once in their lifetime [1] and a smaller subset (6-9\%) will experience recurrent disease (RVVC) $[2,3]$. In the United States and Europe, VVC is predominantly caused by Candida albicans ( 76-89\%) although non-albicans Candida species comprise larger proportions in other geographical regions [4]. Some studies suggest that the abundance of non-albicans Candida has increased over time in VVC infections $[5,6]$, particularly in recurrent disease $[5,7,8]$. Azoles are the standard of care for treatment of VVC; however, increasing rates of azole resistance (in both C. albicans and non-albicans Candida species, such as Candida glabrata, which exhibits higher rates of azole resistance, and Candida krusei, which is intrinsically azole-resistant) threaten the efficacy of this class $[9,10]$. Furthermore, there are concerns regarding risk of relapse, drug-drug interactions, and safety during pregnancy for fluconazole (FLU) [11-13]. There are currently no FDAapproved therapeutics for RVVC and no novel agents have been marketed for VVC in more than 20 years, despite poor outcomes and unmet needs with current therapeutics [14,15].

Echinocandins have been used as standard of care therapy for invasive and bloodstream Candida infections given their excellent safety profile and fungicidal mechanism of action which contribute to their high levels of efficacy clinically [16]. However, limited chemical stability has prevented currently approved echinocandins from topical formulation for use in indications such as VVC. Rezafungin (RZF, formerly CD101) is a novel echinocandin with antifungal potency and spectrum similar to currently approved echinocandins (caspofungin, micafungin, and anidulafungin) [17-19] in 
TABLE 1: MIC values for time-kill assay Candida strains in VSM (pH 4.2) or RPMI (pH 7.0).

\begin{tabular}{|c|c|c|c|c|c|c|c|c|c|c|}
\hline \multirow{3}{*}{ Species } & \multirow{3}{*}{ Strain } & \multirow{3}{*}{$\mathrm{FLU}(\mathrm{S} / \mathrm{R})^{2}$} & \multicolumn{8}{|c|}{$\operatorname{MIC}(\mu \mathrm{g} / \mathrm{ml})^{1}$} \\
\hline & & & \multicolumn{2}{|c|}{ RZF } & \multicolumn{2}{|c|}{ TER } & \multicolumn{2}{|c|}{ FLU } & \multicolumn{2}{|c|}{ AMB } \\
\hline & & & VSM & RPMI & VSM & RPMI & VSM & RPMI & VSM & RPMI \\
\hline \multirow{3}{*}{ C. albicans } & ATCC 44858 & $S$ & 0.06 & 0.03 & 2 & 32 & 1 & 1 & 1 & 0.125 \\
\hline & DPL001 & $\mathrm{R}$ & 0.03 & 0.06 & 64 & 32 & 128 & $>128$ & 0.5 & 0.125 \\
\hline & R357 & $\mathrm{R}$ & 0.5 & 0.25 & 4 & 32 & 0.5 & $>128$ & 0.5 & 0.25 \\
\hline \multirow{3}{*}{ C. glabrata } & CG01 & $S$ & 0.03 & 0.06 & 8 & 0.015 & 16 & 1 & 1 & 0.5 \\
\hline & ATCC 200918 & $\mathrm{R}^{3}$ & 0.125 & 0.06 & $>128$ & 1 & $>128$ & 32 & 1 & 0.5 \\
\hline & MMX 7070 & $\mathrm{R}$ & 0.25 & 0.03 & $>128$ & 2 & $>128$ & 64 & 0.5 & 0.25 \\
\hline \multirow{3}{*}{ C. tropicalis } & СТ02 & $S$ & 0.06 & 0.06 & 4 & 4 & 2 & 0.5 & 1 & 0.25 \\
\hline & MMX 7255 & $\mathrm{R}$ & 0.125 & 0.03 & $>128$ & 64 & $>128$ & 64 & 0.5 & 0.5 \\
\hline & MMX 7525 & $\mathrm{R}$ & 0.125 & 0.06 & $>128$ & 4 & $>128$ & 128 & 0.5 & 0.5 \\
\hline \multirow{3}{*}{ C. parapsilosis } & СР02 & $S$ & 2 & 2 & 4 & 0.03 & 2 & 0.25 & 1 & 0.25 \\
\hline & СР01 & $\mathrm{R}$ & 2 & 2 & 32 & 0.125 & $>128$ & 16 & 1 & 0.25 \\
\hline & MMX 7370 & $\mathrm{R}$ & 2 & 1 & 32 & 0.5 & 64 & 64 & 1 & 0.5 \\
\hline \multirow{2}{*}{ C. krusei } & ATCC 6258 & $\mathrm{R}$ & 0.125 & 0.06 & 16 & 0.5 & 64 & 32 & 1 & 0.5 \\
\hline & ATCC 14243 & $\mathrm{R}$ & 0.06 & 0.06 & 32 & 0.5 & 64 & 32 & 1 & 0.5 \\
\hline
\end{tabular}

${ }^{1}$ MIC values were determined three times, independently. At most there was a 2 -fold range in variation for each drug/strain combination MIC value between replicates and modal values are listed. ${ }^{2}$ Susceptibility to FLU as defined per CLSI $24 \mathrm{~h}$ broth microdilution interpretive criteria [21]. ${ }^{3}$ ATCC describes strain 200918 as FLU-R strain although the CLSI MIC value of $32 \mu \mathrm{g} / \mathrm{ml}$ derived in this study is technically characterized as "susceptible-dose dependent" per CLSI interpretive criteria [21].

addition to a remarkable chemical and metabolic stability that enables topical formulations. A prior study demonstrated that in vitro antifungal activity of RZF was largely retained under low $\mathrm{pH}$ in standard fungal susceptibility testing media against clinical VVC isolates [20]. Whether CD101 fungicidal killing kinetics observed at neutral $\mathrm{pH}$ are also retained in the vaginal environment is unknown.

The purpose of this study was to evaluate the in vitro fungicidal activity of RZF through analysis of time-kill kinetics for FLU-susceptible and FLU-resistant Candida species under conditions and at a $\mathrm{pH}$ relevant to the vaginal microenvironment, using the topical azole VVC therapeutic, terconazole (TER), as a comparator.

\section{Materials and Methods}

2.1. Strains and Culture Conditions. The fourteen Candida strains evaluated in this study included C. albicans ATCC 44858, C. glabrata ATCC 200918, C. krusei ATCC 6258 and 14243 (American Type Culture Collection, ATCC; Chantilly, VA), C. glabrata MMX 7070, Candida parapsilosis MMX 7370, Candida tropicalis MMX 7255 and 7525 (Micromyx, LLC; Kalamazoo, MI), C. glabrata CG01, C. parapsilosis CP01 and CP02, Candida tropicalis CT02 (Wayne State University; Detroit, MI), C. albicans DPL001 (Rutgers University; Newark, NJ), and C. albicans R357 (Eurofins Panlabs, Inc.; St. Charles, MO) (Table 1). For each of the five Candida species evaluated, one fluconazole-susceptible (FLU-S) and two fluconazole-resistant (FLU-R) isolates were chosen, as classified per Clinical and Laboratory Standards Institute (CLSI) interpretive criteria [21], with the exception of $C$. krusei which is intrinsically azole-resistant. Where possible, VVC clinical isolates were included. All strains were cultured aerobically at $35^{\circ} \mathrm{C}$ on Sabouraud dextrose agar (SDA) plates prior to use in susceptibility and time-kill assays.

2.2. Antifungal Agents. Stocks of RZF (Cidara Therapeutics) were prepared fresh in 100\% dimethyl sulfoxide (DMSO; Sigma, cat. number 276855) prior to use. Comparator antifungals, FLU (Alpha Aesar, cat. number J62015), TER (Fluka, cat. number 32355), and amphotericin B (AMB; Sigma, cat. number A2411) were also prepared in 100\% DMSO according to CLSI guidelines [21].

2.3. Antifungal Susceptibility Testing. Susceptibility testing of Candida strains was performed using RPMI 1640 broth (MP Biomedicals, cat. number 1060124) that was buffered with $0.165 \mathrm{M}$ 3-( $N$-morpholino) propanesulfonic acid (MOPS) and then adjusted to $\mathrm{pH} 7.0$ with $1 \mathrm{~N} \mathrm{NaOH}$ or in vaginasimulative medium (VSM) [22]. VSM was prepared with $3.5 \mathrm{~g}$ of $\mathrm{NaCl} / 1,1.4 \mathrm{~g}$ of $\mathrm{KOH} / 1,0.22 \mathrm{~g}$ of $\mathrm{Ca}(\mathrm{OH})_{2} / 1,18 \mathrm{mg}$ of bovine serum albumin/1, $2.2 \mathrm{~g}$ of $90 \%$ lactic acid/l, $1 \mathrm{~g}$ of glacial acetic $\mathrm{acid} / \mathrm{l}, 0.32 \mathrm{~g}$ of $50 \%$ glycerol/1, $0.4 \mathrm{~g}$ of urea/l, $5 \mathrm{~g}$ of glucose $/ \mathrm{l}$, and $6.7 \mathrm{~g}$ of yeast nitrogen base/l and then $\mathrm{pH}$-adjusted to 4.2 using concentrated $\mathrm{HCl}$. Minimal inhibitory concentration (MIC) assays were conducted via broth microdilution in accordance with CLSI guidelines [21,23] with the exception that test compounds were made up at 50x final assay concentration and $100 \mu \mathrm{l}$ assay volumes were used ( $2 \mu \mathrm{l}$ compound stock added to $98 \mu \mathrm{l}$ of broth containing cells at $0.5-2.5 \times$ $10^{3}$ colony-forming units $[\mathrm{CFU}] / \mathrm{ml}$ ). MIC plates were read following a 24 -h incubation at $35^{\circ} \mathrm{C}$ (or $48 \mathrm{~h}$ for some slower growing mutants) and MIC values are reported as the lowest concentrations resulting in prominent growth inhibition ( $50 \%$ ), as specified by CLSI for echinocandins and azoles or at complete inhibition for AMB [23]. Enumeration of MIC assay 

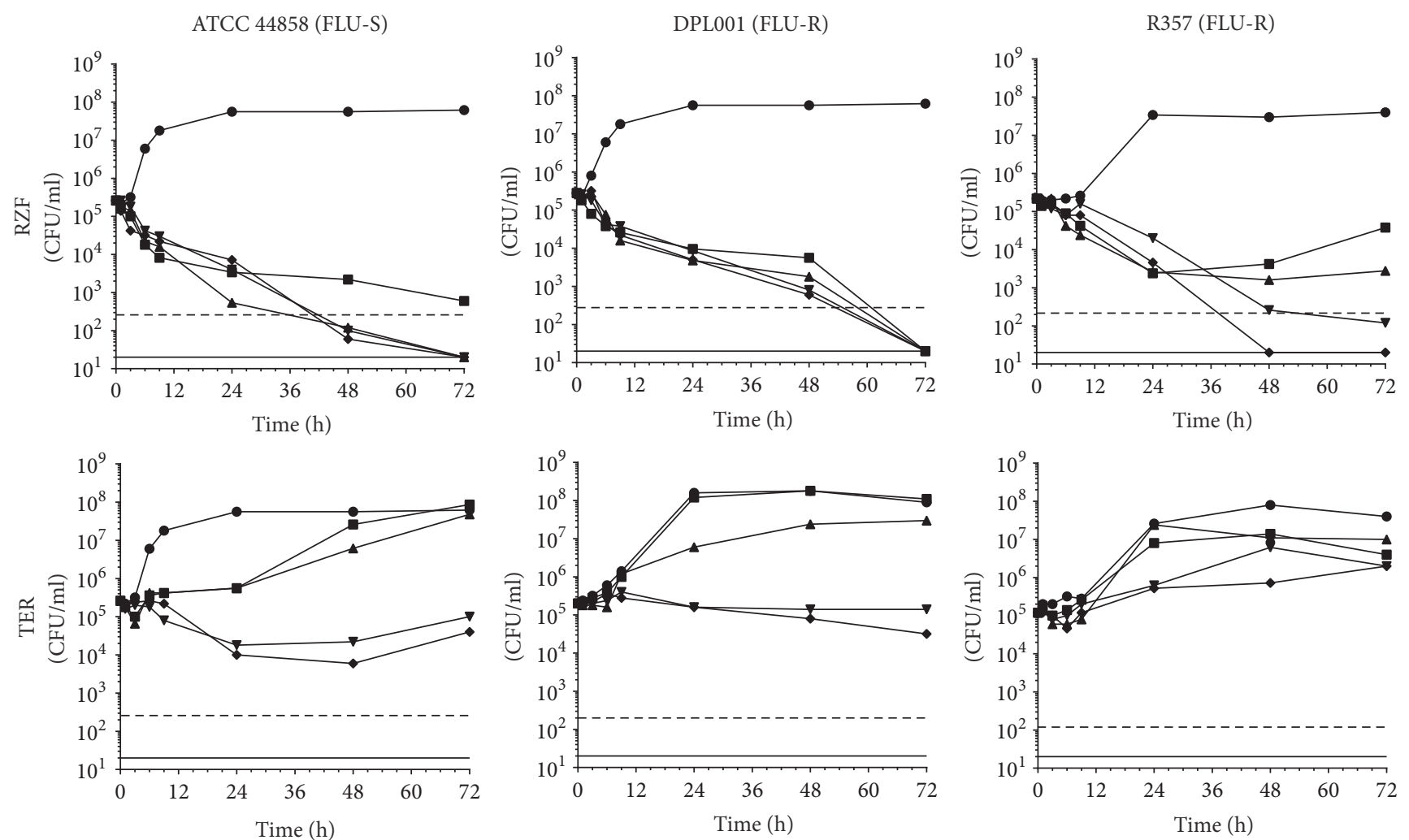

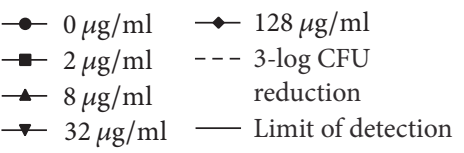

FIgure 1: C. albicans time-kill curves for RZF and TER.

inoculum viable count was performed by plating $50 \mu$ of the starting assay inoculum on SDA. The full MIC panel was run three times, independently. MIC values for each drug/strain combination fell within 2 -fold between replicates and modal MIC values are reported.

2.4. Quality Control. C. krusei ATCC 6258 was used as one of the representative C. krusei strains selected for this study and as a QC strain in susceptibility testing under standard CLSI conditions with AMB and FLU and for comparison to established CLSI QC ranges [21].

2.5. Time-Kill Assays. Time-kill assays were performed as previously described [24]. Overnight SDA cultures of each strain were resuspended in $0.85 \% \mathrm{NaCl}$ to $\sim 1.0 \mathrm{OD}_{530}$ and added to $10 \mathrm{ml}$ VSM to achieve an inoculum of $1.4 \times 10^{5}-3.8 \times$ $10^{5} \mathrm{CFU} / \mathrm{ml}$. Drugs were then added to the inoculated VSM to obtain concentrations of $0,2,8,32$, and $128 \mu \mathrm{g} / \mathrm{ml}$. Timekills were performed in baffled, ventilated $125 \mathrm{ml}$ Erlenmeyer flasks (TriForest, cat. number FBC0125S) incubated at $35^{\circ} \mathrm{C}$ in a shaking incubator. Aliquots were removed from each flask at $0,1,3,6,9,24,48$, and $72 \mathrm{~h}$ and were pelleted/washed twice in $0.85 \% \mathrm{NaCl}$ to remove residual drug and then serially diluted in $0.85 \% \mathrm{NaCl}$. Fifty microliters of each serial dilution was spread onto SDA plates and incubated for $24 \mathrm{~h}$ at $35^{\circ} \mathrm{C}$ (assay limit of detection: $20 \mathrm{CFU} / \mathrm{ml}$; denoted by solid lines in Figures 1-5). Colonies were counted and $\mathrm{CFU} / \mathrm{ml}$ values were calculated and plotted using GraphPad Prism 6 (GraphPad Software, Inc.). Fungistatic activity was defined by CFU reductions $<3$-logs from the starting inoculum, and fungicidal activity was defined by CFU reductions $\geq 3$-logs (denoted by dashed lines in Figures 1-5). Time-kills for each strain-drug combination were performed a single time.

\section{Results}

3.1. Susceptibility Testing. To establish baseline MIC values for RZF and azole comparators in VSM, CLSI broth microdilution values were generated for each Candida strain in both VSM at $\mathrm{pH} 4.2$ and under standard CLSI test media and RPMI at pH 7.0 (Table 1). With the exception of C. parapsilosis (a Candida species with higher intrinsic echinocandin MIC values) RZF was 8- to $>1,024$-fold more potent than TER against all strains tested, in both media types (median 64-fold more potent). Under standard CLSI testing conditions in RPMI media, the 10 strains previously characterized as FLU-R by their respective sources maintained that phenotype, except for C. glabrata ATCC 200918 which came in one dilution under the "resistant" threshold, falling into the "susceptibledose-dependent" designation [21]. In VSM media, RZF MIC 

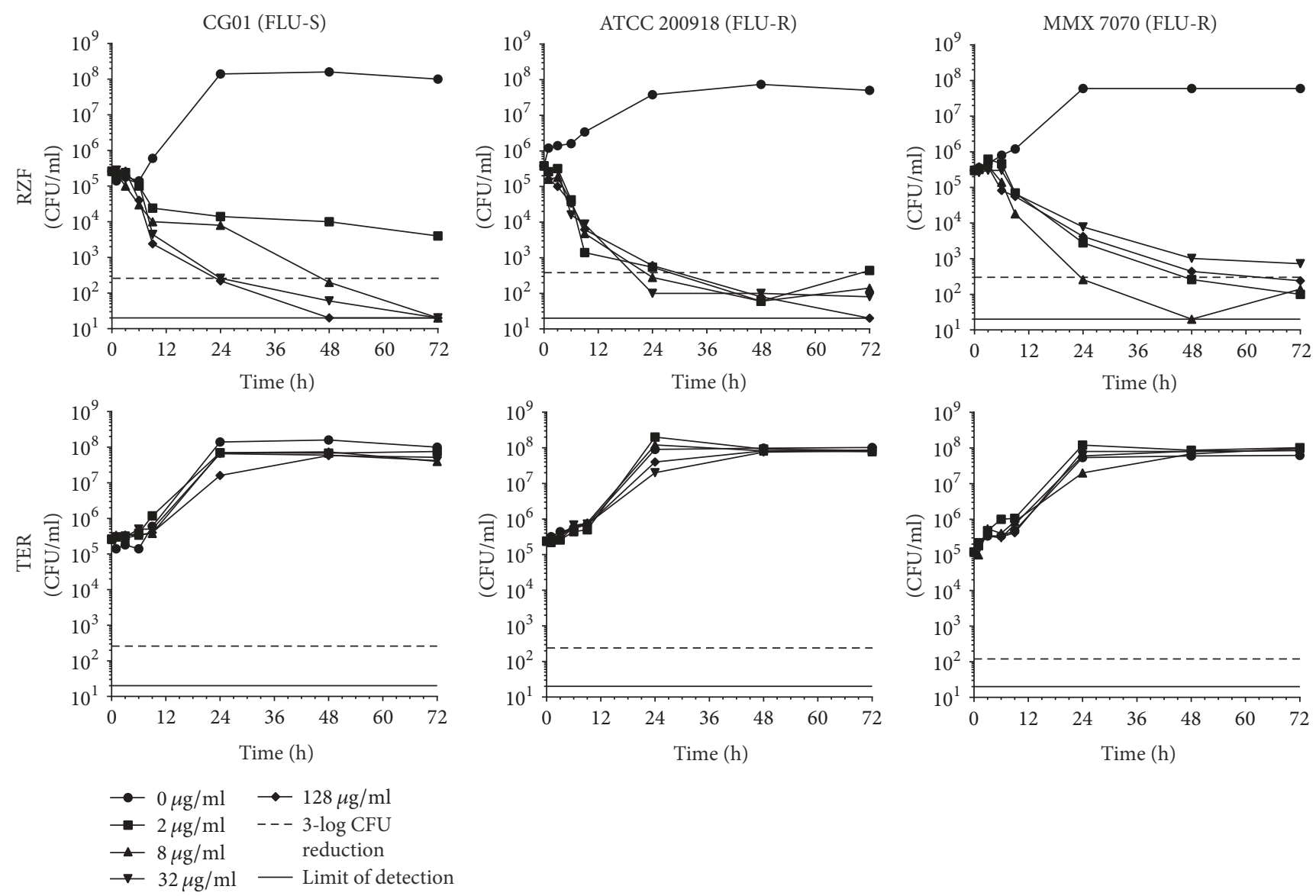

FIgURE 2: C. glabrata time-kill curves for RZF and TER.

values increased 2-fold (median) overall for RZF (range: 1to 8-fold less potent), similarly to AMB. Between the azoles, median TER MIC values were 64-fold higher in VSM than for RPMI (range: 16 -fold more potent to 512-fold less potent) while median FLU MIC values were $\sim 3$-fold less potent (range: $>256$-fold more potent to 16 -fold more potent than in RPMI).

3.2. Quality Control. QC strains and susceptibility criteria are not available for MIC assays performed in VSM; however $\mathrm{pH}$ 7.0 RPMI MIC values derived for FLU and AMB for CLSI QC strain C. krusei ATCC 6258 fell within the established QC ranges (Table 1) [21].

3.3. Time-Kill Kinetics. A high-level summary of log-fold reductions in CFU for each strain/drug combination at $72 \mathrm{~h}$ is presented in Table 2 and individual time-kill curves are shown in Figures 1-5.

(i) C. albicans. RZF was fungicidal against all three C. albicans strains in a dose-dependent manner across the majority of concentrations tested (Figure 1). TER was fungistatic against all strains for which the largest effect observed versus the FLU-S strain ATCC 44858 was a 1 .6-log-fold decrease in $\mathrm{CFU}$ with the highest drug concentration tested $(128 \mu \mathrm{g} / \mathrm{ml})$. (ii) C. glabrata. C. glabrata strains were killed in a dosedependent fungicidal manner by RZF across most concentrations tested, similarly to C. albicans (Figure 2). TER had limited inhibitory activity against all three strains with all concentrations tested resulting in $\sim 3$-logs of growth by $72 \mathrm{~h}$, which was comparable to the no-drug control groups.

(iii) C. tropicalis. Fungicidal activity was observed for RZF versus FLU-S C. tropicalis CT02 by $72 \mathrm{~h}$ at 32 and $128 \mu \mathrm{g} / \mathrm{ml}$ (Figure 3). Against FLU-R C. tropicalis strain MMX 7255, RZF was fungistatic at all concentrations tested (nearfungicidal for $128 \mu \mathrm{g} / \mathrm{ml}$ and $\sim 2$-log-fold reductions for the other three concentrations). For FLU-R C. tropicalis strain MMX 7525, RZF was fungicidal by $72 \mathrm{~h}$ at all concentrations. At earlier time points $(9,24$, and $48 \mathrm{~h})$, the 2 and $8 \mu \mathrm{g} / \mathrm{ml}$ groups generated greater log-fold-reductions in CFU than did the 32 and $128 \mu \mathrm{g} / \mathrm{ml}$ groups. TER was fungistatic against all three $C$. tropicalis strains. The highest activity was observed at 32 and $128 \mu \mathrm{g} / \mathrm{ml}$ versus the CT02 FLU-S strain, resulting in stasis or $\sim 1-\log$ CFU reductions, respectively. Little to no inhibitory activity was observed at the lower TER concentrations against this FLU-S strain or at any concentrations versus the two FLU-R strains.

(iv) C. parapsilosis. RZF was fungistatic against C. parapsilosis CP01 and MMX 7370 at all concentrations tested and was able 

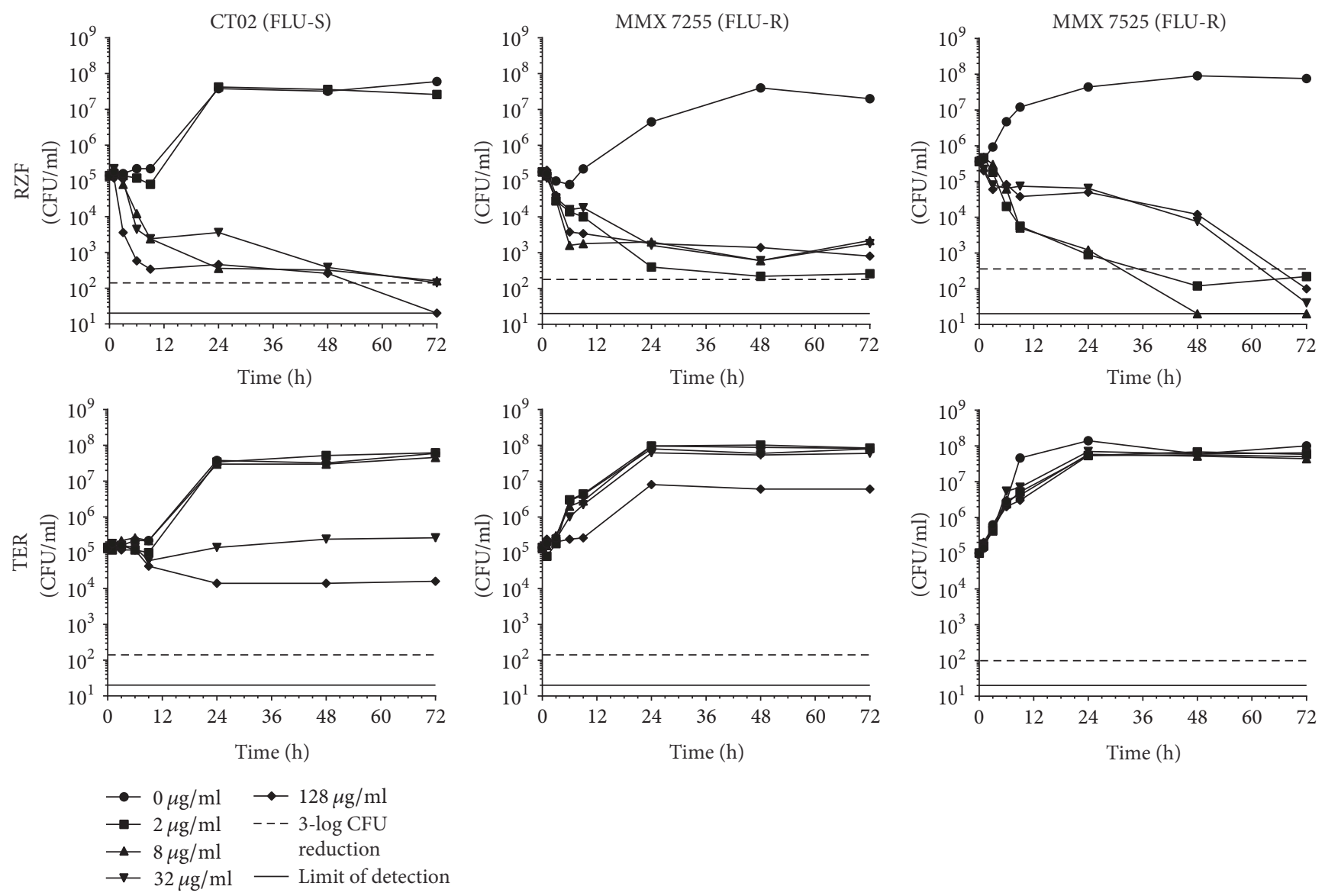

FIgURe 3: C. tropicalis time-kill curves for RZF and TER.

to generate $\mathrm{a} \geq 3-\log \mathrm{CFU}$ reduction against CP02 at $128 \mu \mathrm{g} / \mathrm{ml}$ (Figure 4). Against all three strains, RZF exposure resulted in reductions in CFU by $72 \mathrm{~h}$ at all concentrations except $2 \mu \mathrm{g} / \mathrm{ml}$, where growth occurred. TER produced a similar pattern of killing profile over time to that of RZF versus CP02 but, unlike RZF, TER did not achieve 3-log cidality at any concentration including the highest tested $(128 \mu \mathrm{g} / \mathrm{ml})$. For the two FLU-R C. parapsilosis strains, by $72 \mathrm{~h}$, the 32 and $128 \mu \mathrm{g} / \mathrm{ml}$ TER concentrations resulted in CFU stasis while growth was observed at 2 and $8 \mu \mathrm{g} / \mathrm{ml}$.

(v) C. krusei. RZF demonstrated rapid killing down to the assay limit of detection by $9 \mathrm{~h}$ against both strains of C. krusei and maintained these reductions through all remaining time points (Figure 5), at all concentrations. TER demonstrated fungicidal activity later, at $24 \mathrm{~h}$, and only at the $128 \mu \mathrm{g} / \mathrm{ml}$ concentration. At $32 \mu \mathrm{g} / \mathrm{ml}$, TER generated minor reductions in CFU or stasis, and growth was observed for both strains at 2 and $8 \mu \mathrm{g} / \mathrm{ml}$.

\section{Discussion}

This study demonstrated that RZF anti-Candida and fungicidal activity was retained under in vitro conditions relevant to topical, vaginal application through analysis in MIC assays and evaluation of time-kill kinetics. Furthermore, RZF demonstrated greater potency and fungicidal activity against a variety of Candida species than did TER.

The 5 species included in this study are representative of the most common Candida etiological pathogens of VVC [4, 8 ]. Where possible, clinical vaginal isolates were used (CG01, CP01, CP02, and CT02), although RZF MIC values derived herein and in prior MIC studies do not show a significant difference between strains of vaginal versus nonvaginal origin $[20,25]$. In this study, MIC assays demonstrated that the anti-Candida activity of RZF under conditions simulating the vaginal environment (VSM pH 4.2) was minimally affected compared to its activity in media more closely mimicking systemic administration (RPMI pH 7.0). This retention of activity for RZF in VSM at $\mathrm{pH} 4.2$ was similar to previous observations for RZF in RPMI media at pH 4.0 [20]. Some TER MIC values were lower in VSM than RPMI (C. albicansonly), but the majority were significantly higher in VSM and all TER MIC values in VSM were higher than the corresponding RZF MIC values in VSM. These data are consistent with analysis of azole agents versus VVC isolates across a range of $\mathrm{pH}$ values (4-7) in RPMI media [26, 27].

The test concentrations of RZF and TER of up to $128 \mu \mathrm{g} / \mathrm{ml}$ in MIC and time-kill assays were consistent with the high local concentrations achievable by topical administration, although such drug concentrations presented a challenge and required modification of traditional time-kill 

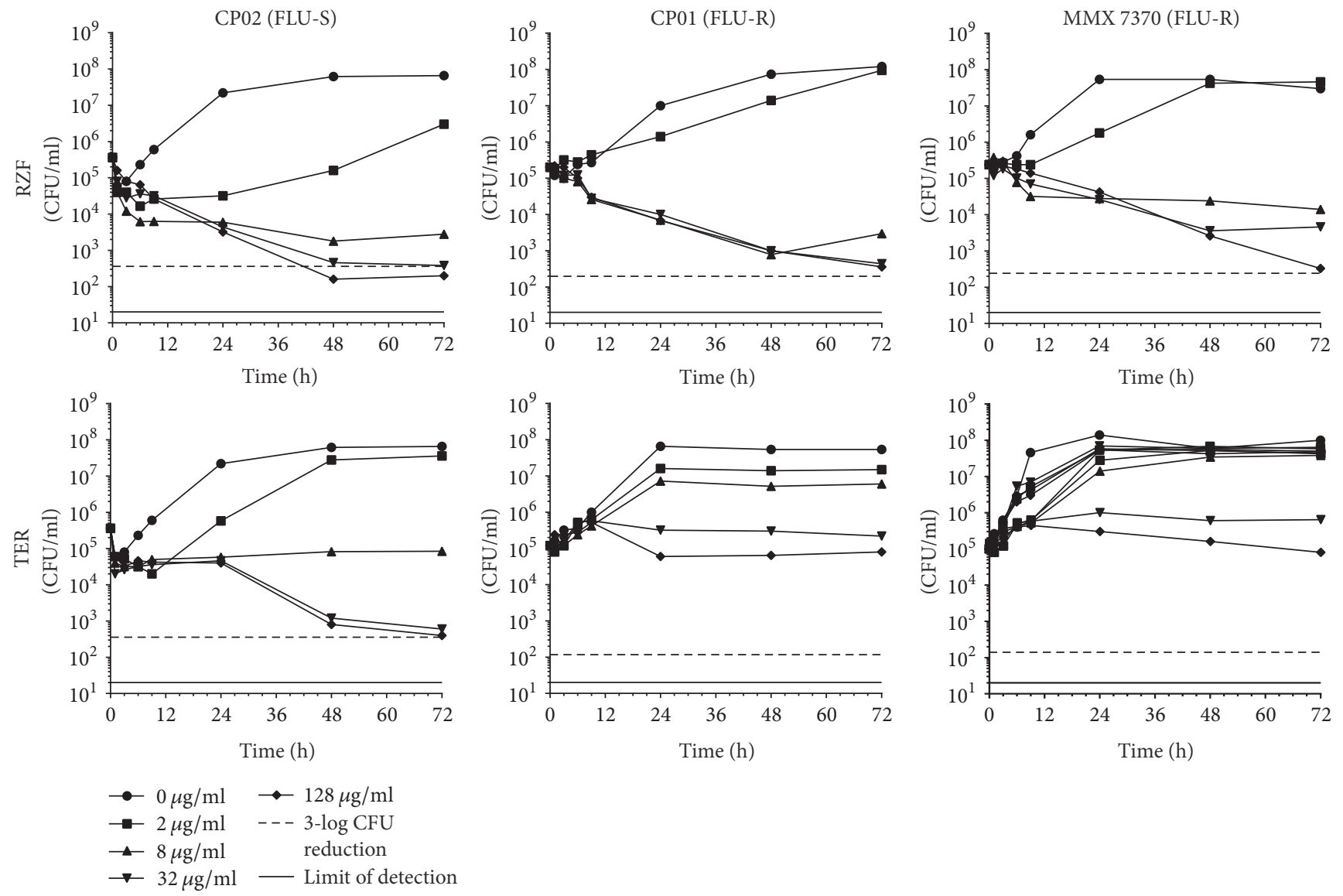

FIgURE 4: C. parapsilosis time-kill curves for RZF and TER.

methodology. Pilot time-kill studies revealed that use of these concentrations resulted in growth inhibition of surviving colonies due to residual drug carried over in the plating aliquots, so a wash step was incorporated into the protocol prior to CFU plating on agar media. Drug release rates of the RZF ointment and gel formulations prepared for clinical evaluation are $1 \%$ and $60 \%$, respectively [28]. Administration of $4 \mathrm{ml}$ of a $6 \% \mathrm{w} / \mathrm{w}$ ointment formulation (gel formulation: $3 \% \mathrm{w} / \mathrm{w})$ at a drug release rate of $1 \%$ would deliver an intravaginal concentration of RZF in excess of $128 \mu \mathrm{g} / \mathrm{ml}$. On a more functional level, $128 \mu \mathrm{g} / \mathrm{ml}$ also represents the solubility limit of RZF in RPMI media (unpublished observations). Although release rates and intravaginal concentration data for TER have not been published, these values for the approved 0.4 and $0.8 \%$ cream formulations would likely fall in between those of the RZF gel and ointment formulations with potentially lower intravaginal drug concentrations than RZF gel due to a lower formulation percentage.

In time-kill assays, RZF demonstrated fungicidal activity against 11 of 14 Candida strains tested and was near-cidal against the remaining 3 strains. Of the five Candida spp. evaluated, C. parapsilosis exhibited the most similar killing kinetics between RZF and TER. The lower CFU reductions versus $C$. parapsilosis for RZF compared to the other Candida spp. in VSM are consistent with, although more pronounced than, trends observed at pH 7.0 in RPMI [18].
This killing profile is shared by other echinocandins against C. parapsilosis as well [29]. Also, the relatively increased killing of TER versus $C$. parapsilosis is consistent with enhanced azole activity against this species in time-kill experiments conducted under standard $\mathrm{pH} 7.0$ conditions [30]. Despite higher MIC values and muted killing kinetics, echinocandins demonstrate strong efficacy against systemic infections caused by C. parapsilosis strains in vivo [31]. Whether a similar in vivo efficacy trend with topical RZF would be observed is yet to be determined. C. parapsilosis does comprise a relatively small percentage (typically $<5 \%$ ) of all VVC isolates $[4,8]$, and the killing kinetics of RZF in VSM were fairly comparable to TER which is clinically efficacious as a topical treatment of VVC caused by C. parapsilosis [32].

RZF demonstrated the highest levels of killing versus $C$. krusei across all concentrations within $9 \mathrm{~h}$ in VSM, similar to killing kinetics observed at pH 7.0 in RPMI for C. krusei strains [18]. Echinocandins are fungicidal against all Candida species as compared to azoles which are fungistatic under standard testing conditions [33]. Yet, despite the characteristic fungistatic mechanism of action of the azole class, a study has shown that azoles can demonstrate fungicidal behavior against $C$. albicans under physiological vaginal conditions [22]. The incongruous in vivo efficacy of these agents in VVC is a phenomenon potentially explained by synergistic activity with acetic acid, as was demonstrated with FLU versus $C$. 

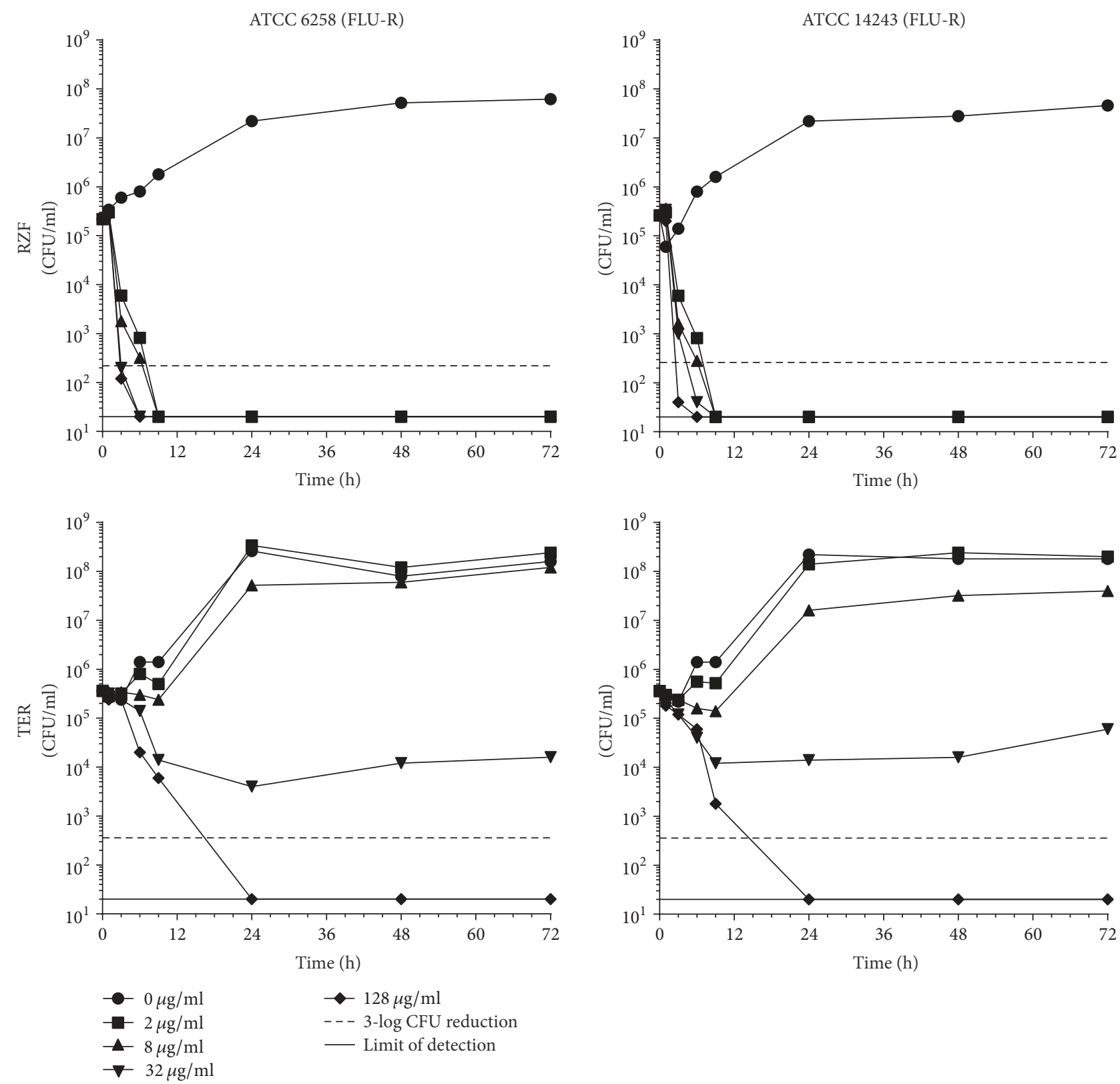

FIgurE 5: C. krusei time-kill curves for RZF and TER.

albicans, C. parapsilosis, C. tropicalis, C. lusitaniae, and C. dubliniensis, however not for C. glabrata or C. krusei [22]. Acetic acid is a component of the VSM used in this study and could be a contributing factor for instances where at least some fungicidal activity towards C. krusei was observed for TER at the highest concentration tested, despite this species being intrinsically resistant to azoles.

A similar incongruity has been observed for RZF, between the susceptibility and time-kill data presented herein as well as strong efficacy data from rat models of VVC [28], and the findings of RADIANT, a Phase 2 clinical trial of two topical formulations of RZF in patients with moderate to severe VVC. Clinical and mycological cures achieved with RZF validated the proof-of-concept of topical echinocandin treatment of acute VVC; however, neither formulation tested in the study generated efficacy data on par with oral fluconazole, and further development of topical RZF was discontinued [34]. Additional work elucidating potential efficacy-limiting factors (e.g., drug distribution, and drug release rates) would be needed to optimize formulations and/or dosing regimens for this indication. Future research may also include characterizing the activity of RZF against Candida biofilms, as recent studies have proposed that biofilms play a role in VVC [35], although their relevance is not entirely known [36]. Finally, the potential impact of native vaginal bacterial flora, such as lactobacilli, on the activity of RZF applied topically could be investigated to rule out any efficacy-hindering interactions. 
TABLE 2: Summary of time-kill log-fold changes in CFU at $72 \mathrm{~h}$.

\begin{tabular}{|c|c|c|c|c|c|c|c|}
\hline \multirow[t]{2}{*}{ Species } & \multirow[t]{2}{*}{ Strain } & \multirow{2}{*}{$\begin{array}{l}\text { Suscept. to FLU } \\
\text { (S/R) }\end{array}$} & \multirow[t]{2}{*}{ Drug } & \multicolumn{4}{|c|}{$\begin{array}{l}\text { Log-fold change in CFU at each drug conc. } \\
\qquad(\mu \mathrm{g} / \mathrm{ml})\end{array}$} \\
\hline & & & & 2 & 8 & 32 & 128 \\
\hline \multirow{6}{*}{ C. albicans } & \multirow{2}{*}{ ATCC 44858} & \multirow{2}{*}{ S } & RZF & ++ & $++++^{*}$ & $++++^{*}$ & $++++^{*}$ \\
\hline & & & TER & - & - & + & + \\
\hline & \multirow{2}{*}{ DPL001 } & \multirow{2}{*}{$\mathrm{R}$} & RZF & $++++^{*}$ & $++++^{*}$ & $++++^{*}$ & $++++^{*}$ \\
\hline & & & TER & - & - & - & - \\
\hline & \multirow{2}{*}{ R357 } & \multirow{2}{*}{$\mathrm{R}$} & $\mathrm{RZF}$ & + & ++ & $+++^{*}$ & $++++^{*}$ \\
\hline & & & TER & - & - & - & - \\
\hline \multirow{6}{*}{ C. glabrata } & \multirow{2}{*}{ CG01 } & \multirow{2}{*}{ S } & RZF & + & $++++^{*}$ & $++++^{*}$ & $++++^{*}$ \\
\hline & & & TER & - & - & - & - \\
\hline & \multirow{2}{*}{ ATCC 200918} & \multirow{2}{*}{$\mathrm{R}$} & $\mathrm{RZF}$ & $-^{*}$ & $+++^{*}$ & $+++^{*}$ & $++++^{*}$ \\
\hline & & & TER & - & - & - & - \\
\hline & \multirow{2}{*}{ MMX 7070} & \multirow{2}{*}{$\mathrm{R}$} & $\mathrm{RZF}$ & $+++^{*}$ & $+++^{*}$ & ++ & $+++^{*}$ \\
\hline & & & TER & - & - & - & - \\
\hline \multirow{6}{*}{ C. tropicalis } & \multirow{2}{*}{ СТ02 } & \multirow{2}{*}{ S } & RZF & - & ++ & $+++^{*}$ & $+++^{*}$ \\
\hline & & & TER & - & - & - & + \\
\hline & \multirow{2}{*}{ MMX 7255} & \multirow{2}{*}{$\mathrm{R}$} & RZF & ++ & + & ++ & ++ \\
\hline & & & TER & - & - & - & - \\
\hline & \multirow{2}{*}{ MMX 7525} & \multirow{2}{*}{$\mathrm{R}$} & RZF & $+++^{*}$ & $++++^{*}$ & $+++^{*}$ & $+++^{*}$ \\
\hline & & & TER & - & - & - & - \\
\hline \multirow{6}{*}{ C. parapsilosis } & \multirow{2}{*}{ СР02 } & \multirow{2}{*}{ S } & RZF & + & ++ & ++ & $+++{ }^{*}$ \\
\hline & & & TER & - & - & ++ & ++ \\
\hline & \multirow{2}{*}{ СР01 } & $\mathrm{R}$ & RZF & - & ++ & ++ & ++ \\
\hline & & & TER & - & - & - & - \\
\hline & MMX 7370 & $\mathrm{R}$ & RZF & - & + & + & ++ \\
\hline & & & TER & - & - & - & - \\
\hline & ATCC 6258 & $\mathrm{R}$ & RZF & $++++^{*}$ & $++++^{*}$ & $++++^{*}$ & $++++^{*}$ \\
\hline C. krusei & ЛI & 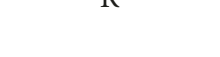 & TER & - & - & + & $++++^{*}$ \\
\hline & ATCC 14243 & $\mathrm{R}$ & RZF & $++++^{*}$ & $++++^{*}$ & $++++^{*}$ & $++++^{*}$ \\
\hline & & & TER & - & - & - & $++++^{*}$ \\
\hline
\end{tabular}

Symbols denote log-fold changes in CFU from starting inoculum: increase or $<1$-log reduction $(-)$; $\geq 1$ - to $<2$-fold reduction $(+)$; $\geq 2$ - to $<3$-fold reduction in $\mathrm{CFU}(++) ; \geq 3$ - to $<4$-fold reduction in CFU $(+++) ; \geq 4$ - to $<5$-fold reduction in CFU $(++++)$. $*$ indicates fungicidal activity ( $\geq 3$-log CFU reduction).

\section{Conclusions}

An echinocandin therapeutic would introduce a novel mechanism of action for VVC, which has predominantly been treated with azole antifungals and remains an area of unmet medical need [2, 37]. The fungicidal activity could offer advantages over azoles in efficacy versus azole-S and azole-R isolates (in particular for C. glabrata). Furthermore, complete eradication of Candida through use of a cidal agent could improve or prevent the risk of recurrent disease. This study demonstrates the retention of anti-Candida activity and fungicidal killing kinetics for RZF under conditions relevant to VVC and at drug concentrations achievable through topical administration. These findings would support future efforts to optimize formulations and dosing strategies to enable translation of these desirable in vitro properties into in vivo efficacy for the treatment and prevention of VVC.

\section{Disclosure}

Portions of this work were previously presented at the 43rd Annual Infectious Disease Society for Obstetrics and Gynecology Meeting (Annapolis, MD, August 11-13, 2016, abstract \#17).

\section{Conflicts of Interest}

Jeffrey B. Locke, Amanda L. Almaguer, Joanna L. Donatelli, and Ken F. Bartizal are employees of Cidara Therapeutics, Inc. Jeffrey B. Locke, Amanda L. Almaguer, and Ken F. Bartizal are shareholders of Cidara Therapeutics, Inc.

\section{Acknowledgments}

The authors thank the following collaborators for their strain contributions: Dr. Jack Sobel (Wayne State University), Drs. 
Chris Pillar and Dean Shinabarger (Micromyx, LLC), and Dr. Lynn Miesel (Eurofins Panlabs, Inc.). Editorial support was provided by Tressa Chung (Scribant Medical) with funding by Cidara Therapeutics, Inc. This study was funded by Cidara Therapeutics, Inc., which had a role in the study design; in the collection, analysis, and interpretation of data; in the writing of this report; and in the decision to submit the article for publication.

\section{References}

[1] J. D. Sobel, S. Faro, R. W. Force et al., "Vulvovaginal candidiasis: epidemiologic, diagnostic, and therapeutic considerations," American Journal of Obstetrics \& Gynecology, vol. 178, no. 2, pp. 203-211, 1998.

[2] B. Foxman, R. Muraglia, J.-P. Dietz, J. D. Sobel, and J. Wagner, "Prevalence of recurrent vulvovaginal candidiasis in 5 European countries and the United States: results from an internet panel survey," Journal of Lower Genital Tract Disease, vol. 17, no. 3, pp. 340-345, 2013.

[3] B. Foxman, R. Barlow, H. D’Arcy, B. Gillespie, and J. D. Sobel, "Candida vaginitis: self-reported incidence and associated costs," Sexually Transmitted Diseases, vol. 27, no. 4, pp. 230-235, 2000.

[4] J. M. Achkar and B. C. Fries, "Candida infections of the genitourinary tract," Clinical Microbiology Reviews, vol. 23, no. 2, pp. 253-273, 2010

[5] A. Spinillo, E. Capuzzo, R. Gulminetti, P. Marone, L. Colonna, and G. Piazzi, "Prevalence of and risk factors for fungal vaginitis caused by non-albicans species," American Journal of Obstetrics \& Gynecology, vol. 176, no. 1, pp. 138-141, 1997.

[6] W. Chaim, M. Mazor, and J. D. Sobel, "Candida albicans vulvovaginitis-trends in care and implications," Harefuah, vol. 133, no. 1-2, pp. 40-43, 1997.

[7] J. D. Mintz and M. G. Martens, "Prevalence of non-albicans Candida infections in women with recurrent vulvovaginal symptomatology," Advances in Infectious Diseases, vol. 03, no. 04, pp. 238-242, 2013.

[8] S. S. Richter, R. P. Galask, S. A. Messer, R. J. Hollis, D. J. Diekema, and M. A. Pfaller, "Antifungal susceptibilities of Candida species causing vulvovaginitis and epidemiology of recurrent cases," Journal of Clinical Microbiology, vol. 43, no. 5, pp. 2155-2162, 2005.

[9] D. Marchaim, L. Lemanek, S. Bheemreddy, K. S. Kaye, and J. D. Sobel, "Fluconazole-resistant candida albicans vulvovaginitis," Obstetrics \& Gynecology, vol. 120, no. 6, pp. 1407-1414, 2012.

[10] Z. Shahid and J. D. Sobel, "Reduced fluconazole susceptibility of Candida albicans isolates in women with recurrent vulvovaginal candidiasis: effects of long-term fluconazole therapy," Diagnostic Microbiology and Infectious Disease, vol. 64, no. 3, pp. 354356, 2009.

[11] D. Mølgaard-Nielsen, H. Svanström, M. Melbye, A. Hviid, and B. Pasternak, "Association between use of oral fluconazole during pregnancy and risk of spontaneous abortion and stillbirth," Journal of the American Medical Association, vol. 315, no. 1, pp. 58-67, 2016.

[12] J. D. Sobel, "Recurrent vulvovaginal candidiasis," American Journal of Obstetrics \& Gynecology, vol. 214, no. 1, pp. 15-21, 2016.

[13] S. Faro, "Systemic vs. topical therapy for the treatment of vulvovaginal candidiasis," Infectious Diseases in Obstetrics and Gynecology, vol. 1, no. 4, pp. 202-208, 1994.
[14] S. Aballéa, F. Guelfucci, J. Wagner et al., "Subjective health status and health-related quality of life among women with Recurrent Vulvovaginal Candidosis (RVVC) in Europe and the USA," Health and Quality of Life Outcomes, vol. 11, no. 1, article 169, 2013.

[15] Y.-X. Zhu, T. Li, S.-R. Fan, X.-P. Liu, Y.-H. Liang, and P. Liu, "Health-related quality of life as measured with the ShortForm 36 (SF-36) questionnaire in patients with recurrent vulvovaginal candidiasis," Health and Quality of Life Outcomes, vol. 14, no. 1, article 65, 2016.

[16] D. S. Perlin, "Current perspectives on echinocandin class drugs," Future Microbiology, vol. 6, no. 4, pp. 441-457, 2011.

[17] M. A. Pfaller, S. A. Messer, P. R. Rhomberg, and M. Castanheira, "Activity of a long-acting echinocandin (CD101) and seven comparator antifungal agents tested against a global collection of contemporary invasive fungal isolates in the SENTRY 2014 Antifungal Surveillance Program," Antimicrobial Agents and Chemotherapy, vol. 61, no. 3, Article ID e02045, 2017.

[18] D. Hall, R. Bonifas, L. Stapert, M. Thwaites, D. L. Shinabarger, and C. M. Pillar, "In vitro potency and fungicidal activity of CD101, a novel echinocandin, against recent clinical isolates of Candida spp." Diagnostic Microbiology and Infectious Disease, vol. 89, no. 3, pp. 205-211, 2017.

[19] M. A. Pfaller, S. A. Messer, P. R. Rhomberg, R. N. Jones, and M. Castanheira, "Activity of a long-acting echinocandin, CD101, determined using CLSI and EUCAST reference methods, against Candida and Aspergillus spp., including echinocandin- and azole-resistant isolates," Journal of Antimicrobial Chemotherapy, vol. 71, no. 10, Article ID dkw214, pp. 2868-2873, 2016.

[20] D. A. Boikov, J. B. Locke, K. D. James, K. Bartizal, and J. D. Sobel, "In vitro activity of the novel echinocandin CD101 at pH 7 and 4 against Candida spp. isolates from patients with vulvovaginal candidiasis," Journal of Antimicrobial Chemotherapy, vol. 72, no. 5, pp. 1355-1358, 2017.

[21] CLSI, M27-S4, Reference Method for Broth Dilution Antifungal Susceptibility Testing of Yeasts, Fourth informational supplement, Clinical and Laboratory Standards Institute, Pennsylvania, Pa, USA, 2012.

[22] M.-Y. S. Moosa, J. D. Sobel, H. Elhalis, W. Du, and R. A. Akins, "Fungicidal activity of Fluconazole against Candida albicans in a synthetic vagina-simulative medium," Antimicrobial Agents and Chemotherapy, vol. 48, no. 1, pp. 161-167, 2004.

[23] CLSI, M27-A3, Reference Method for Broth Dilution Antifungal Susceptibility Testing of Yeasts, Approved Standard, Clinical and Laboratory Standards Institute, Pennsylvania, Pa, USA, 3rd edition, 2008.

[24] M. E. Klepser et al., "Antifungal pharmacodynamic characteristics of fluconazole and amphotericin B tested against Candida albicans," Antimicrobial Agents and Chemotherapy, vol. 41, no. 6, pp. 1392-1395, 1997.

[25] M. A. Pfaller et al., Activity of a Long-Acting Echinocandin (CD101) and Comparator Antifungal Agents Tested Against Contemporary Worldwide Invasive Fungal Isolates, IDWeek, Louisiana, La, USA, 2016.

[26] C. S. Danby, D. Boikov, R. Rautemaa-Richardson, and J. D. Sobel, "Effect of $\mathrm{pH}$ on in vitro susceptibility of Candida glabrata and Candida albicans to 11 antifungal agents and implications for clinical use," Antimicrobial Agents and Chemotherapy, vol. 56, no. 3, pp. 1403-1406, 2012. 
[27] W. Liu et al., "Impact of $\mathrm{pH}$ on the antifungal susceptibility of vaginal Candida albicans," International Journal of Gynecology and Obstetrics, vol. 114, no. 3, pp. 278-280, 2011.

[28] V. Ong et al., "Optimization of CD101 Formulation against Candida albicans in a Rat Model of Vulvovaginal Candidiasis (VVC)," in Proceedings of the ASM Microbe 2016, 2016.

[29] C. J. Clancy, H. Huang, S. Cheng, H. Derendorf, and M. H. Nguyen, "Characterizing the effects of caspofungin on Candida albicans, Candida parapsilosis, and Candida glabrata isolates by simultaneous time-kill and postantifungal-effect experiments," Antimicrobial Agents and Chemotherapy, vol. 50, no. 7, pp. 25692572, 2006.

[30] Y. Li, M. H. Nguyen, H. Derendorf, S. Cheng, and C. J. Clancy, "Measurement of voriconazole activity against Candida albicans, C. glabrata, and C. parapsilosis isolates using time-kill methods validated by high-performance liquid chromatography," Antimicrobial Agents and Chemotherapy, vol. 51, no. 8, pp. 2985-2987, 2007.

[31] M. Fernandez-Ruiz et al., "Initial use of echinocandins does not negatively influence outcome in Candida parapsilosis bloodstream infection: a propensity score analysis," Clinical Infectious Diseases, vol. 58, no. 10, pp. 1413-1421, 2014.

[32] J. L. Thomason, "Clinical evaluation of terconazole. United states experience," J Reprod Med, vol. 34, no. 8 Suppl, pp. 597601, 1989.

[33] G. P. Bodey, "Azole antifungal agents," Clinical Infectious Diseases, vol. 14, pp. S161-S169, 1992.

[34] Cidara Therapeutics Reports Unfavorable Results of Phase 2 RADIANT Trial of CD101 Topical in VVC, 2017, http://ir.cidara .com/phoenix.zhtml?c=253962\&p=irol-newsArticle\&ID=2247615.

[35] C. A. Muzny and J. R. Schwebke, "Biofilms: an underappreciated mechanism of treatment failure and recurrence in vaginal infections," Clinical Infectious Diseases, vol. 61, no. 4, pp. 601606, 2015.

[36] J. D. Sobel, "Editorial Commentary: Vaginal Biofilm: Much Ado about Nothing, or a New Therapeutic Challenge?" Clinical Infectious Diseases, vol. 61, no. 4, pp. 607-608, 2015.

[37] B. Gonçalves, C. Ferreira, C. T. Alves, M. Henriques, J. Azeredo, and S. Silva, "Vulvovaginal candidiasis: Epidemiology, microbiology and risk factors," Critical Reviews in Microbiology, vol. 42, no. 6, pp. 905-927, 2016. 


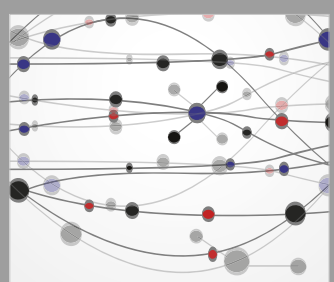

The Scientific World Journal
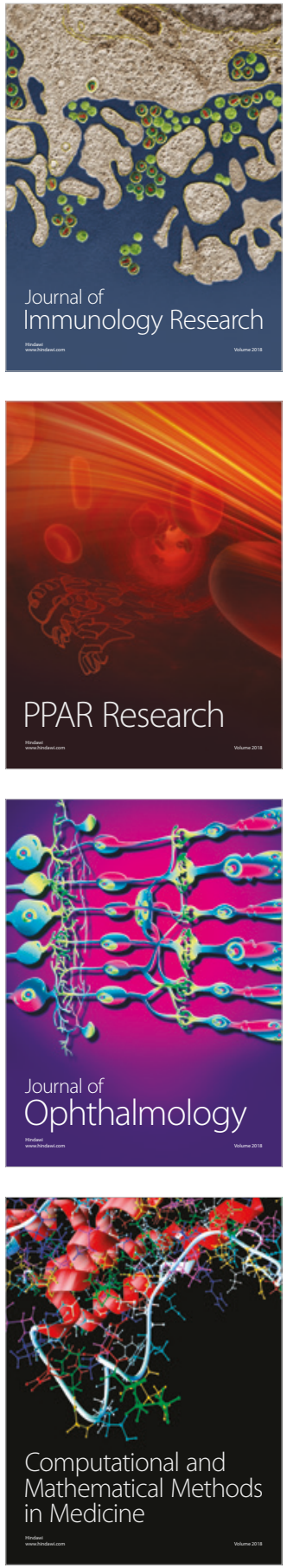

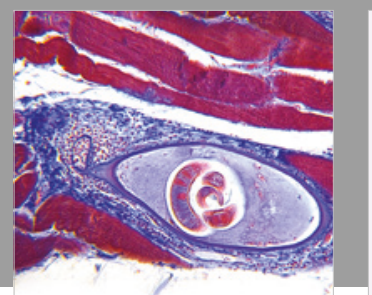

Gastroenterology Research and Practice

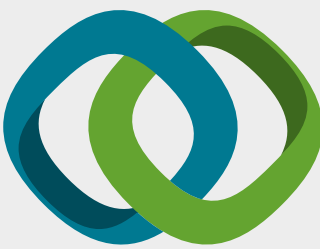

\section{Hindawi}

Submit your manuscripts at

www.hindawi.com
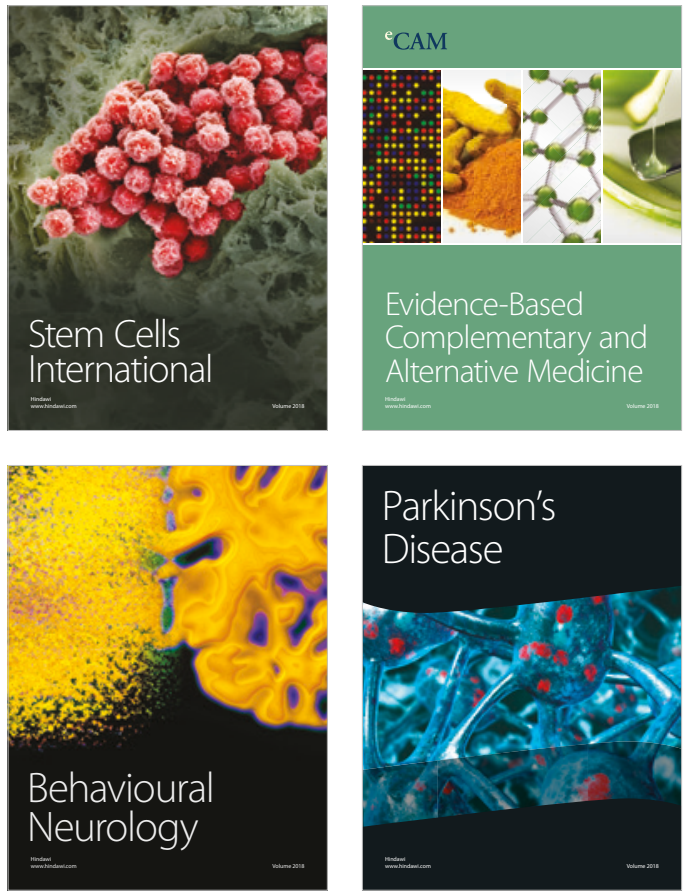

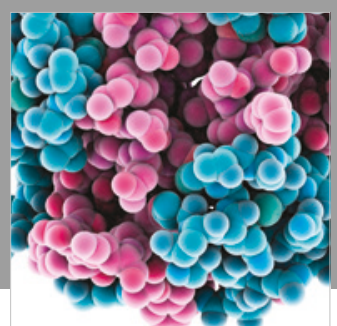

ournal of

Diabetes Research

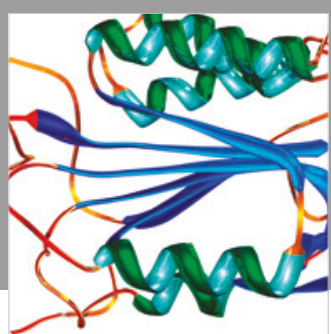

Disease Markers
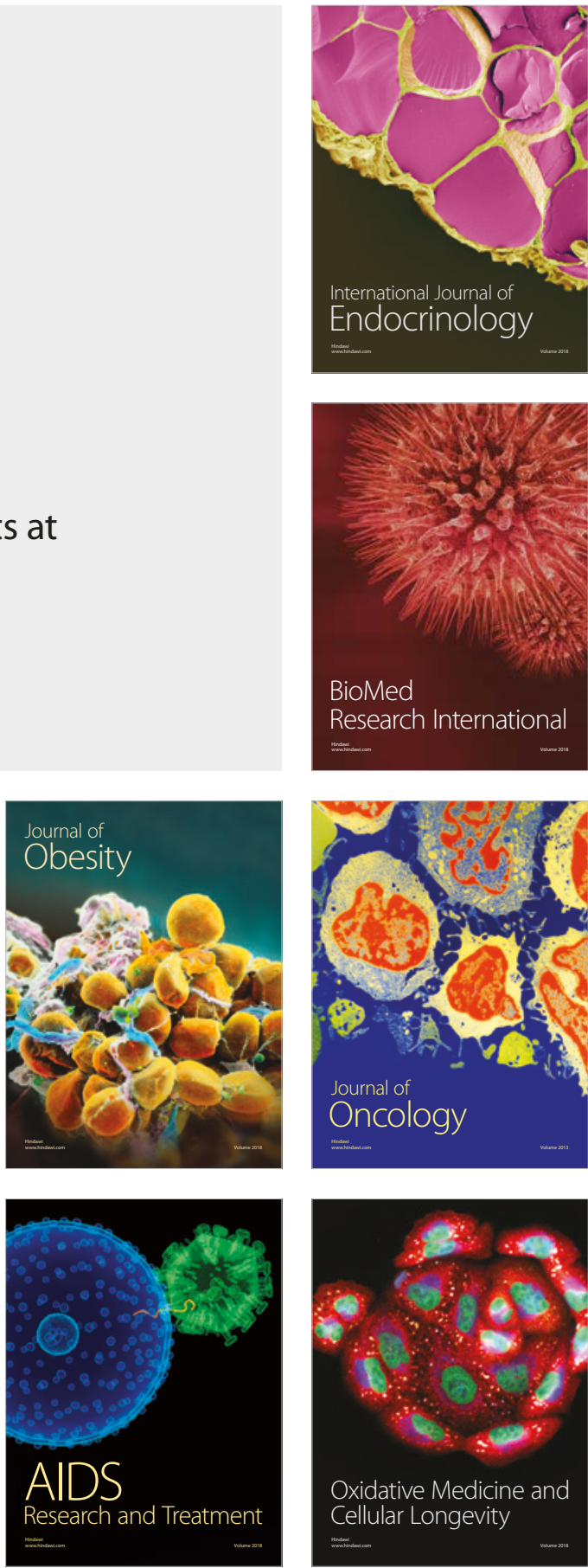